Arch. Tierz., Dummerstorf 43 (2000) 4, 327-335

Aus der Technischen Universitat Munchen, Freising-Weihenstephan, Lehrgebiet fur Tierhaltung und Verhaltenskunde

MARGIT H. ZEITLER-FEICHT und VERENA PRANTNER

\title{
Liegeverhalten von Pferden in Gruppenauslaufhaltung
}

\author{
Herrn Professor Dr. Dr. H. H. Sambraus zum 65. Geburtstag gewidmet
}

\begin{abstract}
Summary
Titel of the paper: Recumbence resting behaviour of horses in loose housing systems with open yards Recumbence resting behaviour was investigated in 48 horses in four loose housing systems with open yards subdivided in areas for feeding, recumbence and for free range. Continuous observations were made for each loose housing system on three independent days during a 24 -hr period.

In all housing systems the horses chose exclusively the area of recumbence to rest in sternal or lateral position. On the one hand the dimension of the area of recumbence influenced the recumbence resting behaviour. In the fourth housing system the insufficient recumbence area reduced the resting time. At the other hand the time of recumbence was dependent on social rank. The animals with lower rank showed a significant shorter recumbence time than the horses with higher rank. However the frequency of recumbence was similar in all horses independent of stable, social rank and age. The results clearly show, that the dimension of the area of recumbence as the only parameter is not enough to satisfy the demand of recumbence in all horses.
\end{abstract}

Key Words: horses, recumbence resting behaviour, loose housing systems

\section{Zusammenfassung}

Das Liegeverhalten von 48 Pferden wurde in vier Offenställen mit getrennten Funktionsbereichen fur Liegen, Fressen und Auslauf uberpruft. Die Beobachtungen erfolgten an drei unabhăngigen Tagen je Stall kontinuierlich über den 24-Stunden-Tag.

Zum Ruhen in der Bauch- und Seitenlage wurde ausschließlich die mit Stroh eingestreute Liegehalle aufgesucht. Dies zeugt von der zentralen Bedeutung der Liegehalle im Offenstall während der kalten Jahreszeit. Die Liegezeiten der Pferde waren einerseits von der Großße der Liegefläche abhăngig. Im Stall mit geringeren Abmessungen als in den Leitlinien zur Pferdehaltung (BMELF, 1995) empfohlen, wurden die kürzesten Liegezeiten festgestellt. Andererseits wurde die Dauer der Liegezeiten entscheidend von der Ranghöhe beeinflußt. Ranghöhere Pferde konnten mehr als doppelt bzw. dreimal so lange in der Bauch- bzw. Seitenlage ruhen als rangniedere. Die Abliegehăufigkeit in einer der beiden Ruhepositionen war unabhăngig vom Betrieb, Rang und Alter bei allen Pferden in etwa gleich hoch. Aus den Befunden ist zu schließen, dass nicht alle Pferde in genügendem Maße ihr Ruhe- bzw. Schlafbedüfnis stillen konnten. Neben einer ausreichend groß bemessenen Liegeflache sind weitere Vorkehrungen zu treffen, um in Offenstallen ein verhaltensgerechtes Ruhen fur alle Pferde zu ermöglichen.

Schlusselworter: Pferde, Liegeverhalten, Gruppenauslaufhaltung

\section{Einleitung}

Gemäß den Leitlinien des BMELF (1995) sollten Pferde, wo immer möglich, in Gruppen gehalten werden. Dabei gilt der Offenlaufstall als die tiergerechteste Haltungsform. In diesem Haltungssystem können die artspezifischen Bedürfnisse der Pferde wie täglich mehrstündige Bewegung, uneingeschränkter Sozialkontakt, vielfältige Umweltreize sowie wahlweiser Aufenthalt unter natürlichen Klimabedingungen weit- 
gehend befriedigt werden. Voraussetzung ist jedoch die richtige Konzeption der Anlage und ein fachgerechtes Management. Fehler in diesen Bereichen erhöhen das Verletzungsrisiko und können erhebliche Beeinträchtigungen einzelner Pferde zur Folge haben. Während Benachteiligungen bei der Futterzuteilung durch entsprechende Fütterungseinrichtungen (PIOTROWSKI, 1989; FLEEGE, 1992; PIRKELMANN, 1997) gemindert werden konnten, fanden mögliche Probleme im Funktionskreis Liegeverhalten bislang noch wenig Beachtung

Ziel vorliegender Untersuchung war deshalb zu überprüfen, ob in Praxisbetrieben mit Gruppenhaltung, die zur Verfügung gestellte Liegefläche von allen Pferden in gleicher Weise zum Liegen in der Bauch- und Seitenlage genutzt werden kann.

2.

2.1 Stallanlage und Tiere

Die Untersuchungen fanden in drei Offenställen (Betrieb B, H und K), die als Pensionspferdehaltungen geführt werden, statt. Das Grundkonzept der Stallanlagen war relativ einheitlich. Jeder Offenstall war in folgende drei Funktionsbereiche untergliedert:

- Überdachter Liegebereich (Tiefstreu mit Stroh)

- Freßbereich mit Freßständen (Heu- und Kraftfuttergabe)

- Auslauf (befestigter und naturbelassener Untergrund)

Jedem Pferd stand eine Gesamtfläche von mindestens $40 \mathrm{~m}^{2}$ zur Verfügung, was den Empfehlungen für Offenstallhaltung mit mindestens $30 \mathrm{~m}^{2} / \mathrm{GV}$ entspricht (ZEITLERFEICHT, 1996; PIRKELMANN, 1997). Ebenso waren die Liegeflächen in den Ställen B bzw. H gemäß den Vorgaben des BMELF (1995) mit mindestens 2,5 x Wh ${ }^{2}$ je Pferd sehr bzw. genügend groß dimensioniert (Tab. 1). Der Stall $\mathrm{K}$ wurde in zwei verschiedenen Situationen (K1 und K2) überprüft. In der Situation K1 war die Liegefläche ebenso wie in $\mathrm{B}$ und $\mathrm{H}$ ausreichend groß bemessen. In der Situation K2 lag die Liegefläche etwa $25 \%$ unter dem empfohlenen Richtwert. Dabei ist zu berücksichtigen, dass das BMELF (1995) eine Reduzierung der Liegefläche um bis zu 20\% toleriert, wenn die Voraussetzungen hinsichtlich Raumstruktur, Pferde und Betreuung günstig sind. Für die Untersuchungen der Situation K1 wurde die Liegefläche von K2 erweitert. Da sich $\mathrm{K} 1$ und $\mathrm{K} 2$ zusätzlich durch einen zum Teil veränderten Tierbestand und eine weitere Flächenänderung im Auslaufbereich unterschieden, werden sie nachfolgend als verschiedene Ställe angeführt.

Tabelle 1

Empfohlene (BMELF, 1995) und vorhandene Liegeflache (LF) in $\mathrm{m}^{2}$ je Stall und $\mathrm{GV}^{*}$ ) (Berechnungsformel: $2,5 \times \mathrm{Wh}^{2 * *}$ je Pferd) (Recommended (BMELF, 1995) and existing area of recumbence (LF) in $\mathrm{m}^{2}$ for each stable and $\mathrm{GV}^{*}$ )

\begin{tabular}{|c|c|c|c|}
\hline Stall & $\begin{array}{c}\text { Anzahl der } \\
\text { Pferde } \\
\text { je Stall }\end{array}$ & $\begin{array}{c}\text { Empfohlene LF/GV in } \mathrm{m}^{2} \text { (BMELF, } \\
1995 \text { ) }\end{array}$ & $\begin{array}{c}\text { Vorhandene LF/GV in } \mathrm{m}^{2} \\
\text { je Stall }\end{array}$ \\
\hline B & 15 & 6,0 & 11,4 \\
\hline $\mathrm{H}$ & 10 & 5,8 & 8,5 \\
\hline K1 & 12 & 5,7 & 7,2 \\
\hline $\mathrm{K} 2$ & 11 & 5,7 & 4,2 \\
\hline
\end{tabular}


Die vier Liegehallen waren überdacht und verfügten jeweils über zwei Ausgänge zum Auslauf bzw. über eine offene Frontseite. Die Liegehallen von $\mathrm{H}$ und $\mathrm{K} 2$ waren nicht strukturiert. In B befand sich im Liegebereich ein Raumteiler und in K1 eine hoch gemauerte Wand. Letztere gliederte die Liegehalle von K1 in zwei Bereiche, wobei zwischen den beiden Abteilungen über einen Durchgang ein Rundlauf möglich war.

Alle Liegehallen waren mit Stroh ausgelegt. Täglich wurde neu eingestreut, wobei die Pferde das frische Stroh auch gerne fraßen. Es gab jedoch keine Strohraufen oder sonstigen Versorgungseinrichtungen in den Liegehallen, ausgenommen in H. Hier war ein Tränkbecken installiert.

Insgesamt waren 48 Pferde in die Untersuchung einbezogen, wobei in jedem Stall mindestens 10 Pferde standen (Tab. 1). Das Alter der Pferde variierte zwischen 2 und 20 Jahren. Alle Tiere, ausgenommen ein Pferd in Stall H, waren zum Zeitpunkt der Untersuchungen bereits seit längerem in ihrer Gruppe integriert. Nach Aussage der Betriebsleiter handelte es sich in jedem Stall um eine untereinander sozial gut verträgliche Gruppe. Die Nutzung der Tiere (Stuten, Wallache) erfolgte ausschließlich im Freizeitbereich. Die Pferde gehörten den verschiedensten Rassen an wie Deutsches Warmblut, Haflinger, Araber und Quarter Horses.

\section{2}

\section{Beobachtungen}

Die Ermittlungen wurden nach Beendigung der Weidesaison von Oktober bis Dezember durchgeführt. Dieser Zeitraum wurde gewählt, um den Einfluß der Jahreszeit auf das Ruheverhalten der Tiere gering zu halten. Die Verhaltensbeobachtungen fanden kontinuierlich über 24-Stunden an jeweils drei unabhängigen Tagen je Stall statt. Sie erfolgten in der Liegehalle und im Freßbereich über Videoaufzeichnungen und zeitgleich im Auslauf über Direktbeobachtungen. Auf diese Weise konnte das Verhalten aller Pferde in der gesamten Offenstallanlage synchron über den 24-Stunden-Tag erfaßt werden. Zur Einzeltiererkennung wurden alle Pferde vor Versuchsbeginn mit Nummern versehen.

Die Merkmale Liegen in der Bauch- und Seitenlage sowie der Aufenthalt in der Liegehalle wurden kontinuierlich erfaßt. Die Beurteilung des Einstreuzustandes der Liegehalle unter hygienischen Aspekten erfolgte für jeden Stall und Beobachtungstag am Morgen und am Abend. Das Wetter wurde im Abstand von sechs Stunden je Stall und Versuchsdurchgang registriert. Die Erfassung der Rangauseinandersetzungen der Pferde je Stall erfolgte, getrennt von den Versuchstagen, anhand mehrtägiger visueller Beobachtungen.

\section{$2.3 \quad$ Auswertung}

Die Rangordnung je Pferd und Stall wurde anhand der Rangindexberechnung nach SAMBRAUS (1975) ermittelt. Dies geschah, indem die Anzahl der Tiere, über die sich ein Individuum überlegen gezeigt hatte, durch die Anzahl der Gruppengenossen, mit denen das Rangverhältnis geklärt war, dividiert wurde. 
Nach dieser Formel liegt der Rangindex ansteigend mit der Ranghöhe stets zwischen 0,0 und 1,0. Ein Rangverhältnis galt nur dann als geklärt, wenn mindestens doppelt so viele dominante Verhaltensweisen das eine Tier von dem anderen unterschied.

Für die varianzanalytische Auswertung kam aus dem SAS-Programm-Paket (Proc. GLM) die Methode Least-Square zum Einsatz.

$\begin{array}{cl}y_{i j k l m n}=\mu+b_{i}+ & r_{j}+a_{k}+w_{1}+t_{m}\left(b_{i}+r_{j}+a_{k}\right)+e_{i j k l m n} \\ y_{i j k l m n} & \text { Merkmalsausprägung } \\ \mu & \text { Mittelwert } \\ b_{i} & \text { fixer Effekt des Stalles }(i=1,2, \ldots, 4) \\ r_{j} & \text { fixer Effekt des Ranges }(j=1,2) \\ a_{k} & \text { fixer Effekt des Alters }(k=1,2) \\ w_{l} & \text { fixer Effekt des Wetters }(1=1,2, \ldots, 4) \\ t_{m} & \text { zufälliger Effekt des Tieres }(m=1,2, \ldots, 48) \\ e_{i j k l m n} & \text { zufälliger Restfehler }\end{array}$

Signifikante Unterschiede $(\mathrm{p} \leq 0,05)$ sind in den Tabellen durch unterschiedliche Buchstaben gekennzeichnet.

Überprüft wurden die Merkmale Liegedauer, getrennt nach Liegezeiten in der Bauchund Seitenlage, die Aufenthaltsdauer in der Liegehalle und die Abliegehäufigkeit in Abhängigkeit von Betrieb (B, H, K1 und K2), Wetter, Rang und Alter. Für letztere wurden jeweils zwei Rang- (R1 und R2) und Altersklassen (A1 und A2) gebildet.

$\mathrm{R} 1: \quad$ Tiere mit Rangindex $\leq 0,5 \quad \mathrm{R} 2: \quad$ Tiere mit Rangindex $>0,5$

A1: $\quad$ Tiere $\leq 7$ Jahre A2: Tiere $>7$ Jahre

Das Wetter wurde mit Sonne, Wolken, Regen und Schnee in vier Kategorien unterteilt. Für den Einstreuzustand der Liegehalle erfolgte eine Klassifizierung mit „trocken“, „überwiegend trocken" und ,überwiegend feucht" in drei Gruppen.

\section{Ergebnisse}

\subsection{Liegebereich und Wetter}

Zum Ruhen in der Bauch- und Seitenlage wurde in allen Ställen stets die Liegehalle aufgesucht. Während der gesamten Beobachtungszeit konnte weder im Freßbereich noch im Auslauf ein Abliegevorgang mit anschließendem Ruhen registriert werden.

Der Einstreuzustand wurde in jedem Stall und Versuchsdurchgang stets mit ,überwiegend trocken" eingestuft. Deshalb konnte für dieses Merkmal keine statistische Auswertung erfolgen.

Das Liegeverhalten der Pferde und ihre Aufenthaltsdauer in der Liegehalle wurden vom Wetter nicht beeinflußt. Die täglichen Wetterschwankungen waren in der kalten Jahreszeit zu gering, um eine Änderung des Ruheverhaltens zu bewirken.

\subsection{Liegezeiten in der Bauch- und Seitenlage}

Die Liegezeit der Pferde in der Bauchlage variierte je Stall zwischen 42,0 $\mathrm{min} / \mathrm{Tag}$ (K2) und 66,8 min/Tag (B) (Tab. 2). Dabei nahmen die Pferde in K2 mit der zu gering 
bemessenen Liegefläche signifikant weniger oft die Bauchlage ein als die Pferde in B mit dem größten Liegeflächenangebot. In letzterem ruhten die Tiere im Vergleich zu denen in $\mathrm{H}$ und $\mathrm{K} 1 \mathrm{mit}$ nur 3,1 min/Tag die kürzeste Zeit in der Seitenlage ( $\mathrm{p} \leq 0,01)$.

Tabelle 2

Least Square-Means (LSM) und Standardfehler (SE) der Liegezeit der Pferde/24-Stunden-Tag (min) in der Bauch- und Seitenlage je Stall (B, H, K1 und K2) (LS means and standard errors for resting time of horses per 24-hr-day $(\mathrm{min})$ in sternal and lateral recumbence depending on the loose housing system with open yard)

\begin{tabular}{lllllllll}
\hline Ruheposition & \multicolumn{3}{c}{ B } & \multicolumn{3}{c}{ H } & \multicolumn{2}{c}{ K1 } \\
& LSM & SE & LSM & SE & LSM & SE & LSM & SE \\
\hline Bauchlage & $66,8 \mathrm{a}$ & $\pm 7,6$ & $59,0 \mathrm{ab}$ & $\pm 9,9$ & $60,6 \mathrm{ab}$ & $\pm 8,3$ & $42,0 \mathrm{~b}$ & $\pm 7,6$ \\
Seitenlage & $3,1 \mathrm{c}$ & $\pm 3,3$ & $27,5 \mathrm{a}$ & $\pm 4,3$ & $17,6 \mathrm{ab}$ & $\pm 3,6$ & $11,4 \mathrm{bc}$ & $\pm 4,0$ \\
\hline
\end{tabular}

Ranghöhere Pferde (R2) konnten mit 80,5 bzw. 22,8 min/Tag deutlich mehr Zeit in der Bauch- bzw. Seitenlage ruhen als rangniedrigere (Tab. 3). Letztere (R1) lagen mit durchschnittlich 33,8 min/Tag weniger als halb solange in der Bauchlage. Noch größer war der Unterschied bezüglich der Seitenlage. Die rangtieferen Tiere verbrachten durchschnittlich nur 6,9 min/Tag in dieser Ruheposition ( $\mathrm{p} \leq 0,001)$.

Auch für das Merkmal Alter ergaben sich signifikante Unterschiede (Tab. 3). Die jüngeren Pferde (A1) lagen mit durchschnittlich 68,8 bzw. 22,7 min/Tag eindeutig mehr Zeit in der Bauch- bzw. Seitenlage als die älteren Tiere (A2) mit 45,5 bzw. 7,00 $\min / \operatorname{Tag}(\mathrm{p} \leq 0,001)$.

Tabelle 3

Least Square-Means (LSM) und Standardfehler (SE) der Liegezeit der Pferde/24-Stunden-Tag ( $\mathrm{min}$ ) in der Bauch- und Seitenlage in Abhängigkeit von Rangordnung (R1, R2) und Alter (A1, A2) (LS means and standard errors for resting time of horses per 24-hr-day ( $\mathrm{min})$ in sternal $(\mathrm{BL})$ and lateral $(\mathrm{SL})$ recumbence depending on rank- and age-classes)

\begin{tabular}{lcccccccc}
\hline Ruheposition & \multicolumn{2}{c}{ R1 } & \multicolumn{2}{c}{ R2 } & \multicolumn{2}{c}{ A1 } & \multicolumn{2}{c}{ A2 } \\
& LSM & SE & LSM & SE & LSM & SE & LSM & SE \\
\hline Bauchlage & $33,8 \mathrm{a}$ & $\pm 5,5$ & $80,5 \mathrm{~b}$ & $\pm 5,4$ & $68,8 \mathrm{a}$ & $\pm 6,4$ & $45,5 \mathrm{~b}$ & $\pm 4,2$ \\
Seitenlage & $6,9 \mathrm{a}$ & $\pm 2,4$ & $22,8 \mathrm{~b}$ & $\pm 2,3$ & $22,7 \mathrm{a}$ & $\pm 2,8$ & $7,0 \mathrm{~b}$ & $\pm 1,8$ \\
\hline
\end{tabular}

Einzeltierergebnisse:

Es konnte ermittelt werden, dass manche Pferde nicht täglich zum Abliegen in eine der beiden Ruhepositionen kamen. Zwei Pferde nahmen an den drei Untersuchungstagen weder die Bauch- noch die Seitenlage ein. Ruhen ohne Seitenlage über einen Zeitraum von drei Tagen wurde insgesamt sechsmal beobachtet.

\subsection{Aufenthaltsdauer in der Liegehalle}

Als Aufenthaltsdauer in der Liegehalle wurde der Zeitraum bezeichnet, den ein Pferd je Tag in diesem Funktionsbereich über die Summe aller Aktivitäten wie Liegen, Strohfressen, Dösen und Stehen verbrachte. In Stall B mit dem größten Platzangebot hielten sich die Pferde mit durchschnittlich 473,1 $\mathrm{min} / \mathrm{Tag}$ mehr als doppelt solange in der Liegehalle auf als in den anderen Ställen ( $p \leq 0,001)$. Dies entspricht einem Anteil

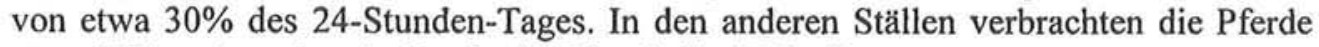
etwa $15 \%$ und weniger je Tag in der Liegehalle (Tab. 4). 
Tabelle 4

Least Square-Means (LSM) und Standardfehler (SE) der Aufenthaltsdauer der Pferde/24-Stunden-Tag (min) in der Liegehalle je Stall (B, H, K1 und K2) (LS means and standard errors for staying time of horses per 24-hr-day in the cubicle house for each loose housing system with open yard)

\begin{tabular}{ccccc}
\hline Aufenthaltsdauer & B & H & K1 & K2 \\
\hline LSM & $473,1 \mathrm{a}$ & $187,9 \mathrm{bc}$ & $242,7 \mathrm{~b}$ & $163,9 \mathrm{c}$ \\
SE & $\pm 21,3$ & $\pm 27,7$ & $\pm 23,2$ & $\pm 25,5$ \\
\hline
\end{tabular}

Die Aufenthaltsdauer in der Liegehalle war nicht vom Alter abhängig (Tab. 5). Die Pferde der Altersklasse A1 befanden sich mit 280,7 min/Tag ähnlich lange im Liegebereich wie die Tiere aus A2 (253,1 min/Tag). Rangniedere Pferde (R1) konnten sich jedoch mit 174,9 $\mathrm{min} / \mathrm{Tag}$ durchschnittlich nur etwa halb solange in der Liegehalle aufhalten als die ranghöheren Tiere der Gruppe R2 mit 358,9 min/Tag ( $\mathrm{p} \leq 0,001)$.

Tabelle 5

Least Square-Means (LSM) und Standardfehler (SE) der Aufenthaltsdauer der Pferde/24-Stunden-Tag (min) in Abhängigkeit von Rangordnung (R1, R2) und Alter (A1, A2) (LS means and standard errors for staying time of horses per 24-hr-day in the cubicle house depending on rank- and age-classes)

\begin{tabular}{ccccc}
\hline Aufenthaltsdauer & R1 & R2 & A1 & A2 \\
\hline LSM & $174,9 \mathrm{a}$ & $358,9 \mathrm{~b}$ & 280,7 & 253,1 \\
SE & $\pm 15,2$ & $\pm 15,0$ & $\pm 17,8$ & $\pm 11,8$ \\
\hline
\end{tabular}

\section{4}

Abliegehäufigkeit in der Liegehalle

Die Abliegehäufigkeit umfaßt die Anzahl der täglichen Abliegevorgänge je Pferd in eine der beiden Ruhepositionen. Unabhängig von Stall, Rang und Alter legten sich die Pferde mit durchschnittlich 1,9 bis 2,6 mal je Tag etwa gleich häufig in die Bauchoder Seitenlage ab (Tab. 6).

Tabelle 6

Least Square-Means (LSM) und Standardfehler (SE) zur Anzahl der Abliegevorgănge/24-Stunden-Tag in Abhängigkeit von Stall (B, H, K1, K2), Rangordnung (R1, R2) und Alter (A1, A,2) (LS means and standard errors for the frequency of recumbence per 24-hr-day ( $\mathrm{min}$ ) depending on the loose housing system with open yard, rankclasses and age-classes)

\begin{tabular}{ccccccccc}
\hline Abliegevorgang & $\mathrm{B}$ & $\mathrm{H}$ & $\mathrm{K} 1$ & $\mathrm{~K} 2$ & $\mathrm{R} 1$ & $\mathrm{R} 2$ & $\mathrm{~A} 1$ & $\mathrm{~A} 2$ \\
\hline LSM & 2,4 & 1,9 & 2,5 & 2,5 & 2,1 & 2,6 & 2,6 & 2,1 \\
SE & $\pm 0,33$ & $\pm 0,43$ & $\pm 0,36$ & $\pm 0,40$ & $\pm 0,24$ & $\pm 0,23$ & $\pm 0,28$ & $\pm 0,18$ \\
\hline
\end{tabular}

4. Diskussion und Schlußfolgerung

Unter naturnahen Bedingungen bevorzugen Pferde zum Liegen einen trockenen Untergrund (KLINGEL, 1972). Der Boden muß jedoch auch eine gewisse „Weichheit" aufweisen, da zu harter Boden, wie Beton oder Rasengittersteine, ebenso wie zu feuchter Boden in der Regel gemieden werden. Auch in vorliegender Untersuchung suchten die Pferde zum Ruhen im Liegen ausschließlich den mit Stroh eingestreuten „weichen“ Liegebereich auf. Dieses Verhalten beweist, dass der Liegehalle in Offenställen in der kalten Jahreszeit als Liegebereich eine zentrale Bedeutung zukommt.

Die Größe der Liegefläche sollte deshalb im Offenstall den Richtwert von mindestens $2,5 \times W^{2}$ je Pferd (BMELF, 1995) keinesfalls unterschreiten. Im Stall K2 mit der geringsten Liegefläche konnten deutlich verkürzte Liegezeiten im Vergleich zu den an- 
deren Ställen beobachtet werden. Einzige Ausnahme waren die auffallend geringen Liegezeiten in der Seitenlage in Stall B, obwohl dieser das größte Flächenangebot je Pferd aufwies und zusätzlich über einen Raumteiler verfügte. Der Befund konnte mit der großen Tierzahl sowie mit der relativ langen Aufenthaltsdauer der Pferde in der Liegehalle in Zusammenhang gebracht werden. Dadurch kam es zu vermehrt Aktivität und Unruhe im Liegebereich. Die Folge war, dass insbesondere rangniedere Tiere immer wieder gezwungen waren, aufzustehen und auszuweichen. Somit war die erforderliche Sicherheit, die adulte Pferde zum Ruhen in der Seitenlage benötigen, in Stall $B$ nicht in ausreichendem Maß gegeben.

Das Deutsche und das Schweizer Tierschutzgesetz fordern für gehaltene Tiere eine verhaltensgerechte Unterbringung. Verhaltensgerecht ist eine Unterbringung jedoch nur dann, wenn die wesentlichen Bedürfnisse der jeweiligen Tierart in jedem Funktionskreis befriedigt werden. Das Schlafbedürfnis ist als essentielles Bedürfnis zu werten, denn Tiefschlaf ist für Säugetiere lebensnotwendig. Dieser ist bei Pferden, entgegen der allgemein verbreiteten Meinung, nur im Liegen in der Bauch- oder Seitenlage möglich wie RUCKEBUSCH (1972) sowie DALLAIRE und RUCKEBUSCH (1974) anhand von EEG- und Muskeltonusmessungen feststellten. Zwar konnten in vorliegender Untersuchung die Pferde, wenn man den Stalldurchschnitt betrachtet, in allen Ställen ausreichend lange Zeit in der Bauch- und Seitenlage verbringen. Die durchschnittlichen Liegezeiten der Tiere je Stall decken sich mit einem Anteil am 24-Stunden-Tag von 4,8\% (Stall B), 6,0\% (Stall H), 5,4\% (Stall K1) und 3,7\% (Stall K2) mit den Werten, die man bei Freilandbeobachtungen gewann. Danach ruhen adulte Pferde zwischen 3 und 11\% des 24-Stunden-Tages im Liegen (KOWNACKI et al., 1978; DUNCAN, 1980; BOYD et al., 1988). Betrachtet man jedoch das Liegeverhalten in Abhängigkeit von der Rangklasse, so zeigt sich eindeutig, dass ranghöhere Tiere mehr als doppelt bzw. dreimal solange in der Bauch- bzw. Seitenlage liegen als rangniedere. Dies entspricht für erstere einem Anteil der Liegezeit am 24-Stunden-Tag von durchschnittlich 7,8\%, für letztere von lediglich $2,8 \%$. Da sich jedoch sowohl die ranghöheren als auch die rangtieferen Pferde mit 2,6 und 2,1 mal je Tag in etwa gleich häufig zum Ruhen ablegten, kann davon ausgegangen werden, dass die unterlegenen Tiere ihr Liege- und vermutlich auch ihr Schlafbedürfnis nicht in ausreichendem Maße stillen konnten.

Betrachtet man die Ergebnisse je Einzeltier, so ist unter dem Aspekt der Tiergerechtheit besonders kritisch zu werten, dass in vorliegender Untersuchung einige Tiere im Verlauf des 24-Stunden-Tages kein einziges Mal zum Abliegen in eine der beiden Ruhepositionen kamen. Der für die körperliche Regeneration erforderliche slow-wavesleep kann jedoch nur im Liegen erfolgen. Ebenso der REM (rapid eye movement) Schlaf mit Traumphasen, der vermutlich der ,psychischen“ Erholung dient. Für ihn ist beim Pferd die Einnahme der Seitenlage notwendig (RUCKEBUSCH, 1972; DALLAIRE und RUCKEBUSCH, 1974). Nach STEINHART (1937) legen sich Pferde unter menschlicher Obhut mindestens einmal pro Tag ab. Das in vorliegender Untersuchung von der Norm abweichende Verhalten einiger Pferde konnte mit der Rangordnung in Zusammenhang gebracht werden. Es waren durchweg rangniedere Tiere davon betroffen. 
Die unterschiedlichen Liegezeiten je Altersklasse entsprechen dem Normalverhalten von Equiden. Danach ist der Anteil der Ruhephasen im Liegen bei jungen Tieren höher als bei älteren (KOWNACKI et al., 1978; DUNCAN, 1980). Besonders die Seitenlage, bevorzugte Ruheposition jüngerer Tiere, wurde von den Pferden mit weniger als sieben Jahren mehr als dreimal solange eingenommen als von den älteren Tieren.

Nach vorliegenden Untersuchungen konnten in Offenlaufställen die Liegeflächen, obwohl deren Abmessungen den Empfehlungen des BMELF (1995) entsprachen, nicht von allen Pferde in gleicher Weise zum Schlafen bzw. zum Ruhen in der Bauch- und Seitenlage genutzt werden. Eindeutig benachteiligt waren vor allem die rangniederen Tiere. Das Bedürfnis sich abzulegen und zu ruhen, dürfte jedoch bei allen Pferden in ähnlicher Weise vorhanden gewesen sein. Darauf deutet die unabhängig vom Rang gleich hohe Anzahl an Abliegevorgängen hin. Daraus folgt, dass für Liegehallen von Offenställen ein ausreichend scheinendes Flächenangebot nicht genügt, um das Liegebedürfnis aller Pferde zu befriedigen, sondern zusätzliche Maßnahmen erforderlich sind. Es gilt zu überprüfen, inwieweit eine Reduzierung der Gruppengröße, Raumteiler oder Sichtblenden etc. helfen, die Voraussetzungen für ein verhaltensgerechtes Ruhen der Pferde zu verbessern. Insbesondere sollte darauf geachtet werden, die Liegehalle als ausschließlichen Ruheraum zu konzipieren. Um das Aufsuchen der Liegehalle zum Strohfressen zu vermeiden, empfehlen sich Sägespäne oder ăhnliche Materialien als Einstreu.

BMELF:

\section{Literatur}

Leitlinien zur Beurteilung von Pferdehaltungen unter Tierschutzgesichtspunkten. Hrsg.: Bundesministerium fur Ernahrung, Landwirtschaft und Forsten (BMELF), Referat Tierschutz, Bonn, 1995

BOYD, L.E.; CARBONARO, A.D.; HOUPT, K.A.:

The 24-hour time budget of przewalski horses. Appl. Anim. Behav. Sci. 21 (1988), 5 - 17

DALLAIRE, A.; RUCKEBUSCH, Y.:

Sleep patterns in the pony with observations in partial perceptual deprivation. Physiol. Behav. 12 (1974), 789 - 796

DUNCAN, P.:

Time-budgets of camargue horses. Behaviour 72 (1980), 26 - 49

FLEEGE, G.:

Verhalten einer Haflingergruppe als Maßstab für eine tiergerechte Futterversorgung im Rahmen der Gruppenauslaufhaltung. Univ. München, Diss. med. vet., 1992

KLINGEL, H.:

Das Verhalten der Pferde (Equidae). In: Handbuch der Zoologie. Hrsg.: HELMCKE J.-G., D. STARK und W. WERMUTH. 10 (1972), 1 - 68

KOWNACKI, M.; SASLMOWSKI, E.; BUDZYNSKI, W.; JEZIERSKI, T.:

Observations of the twenty-four hours rhythm of natural behaviour of polnish primitiv horse bred for conservation of genetic resources in a forest reserve. Genetica Polonica 10 (1978), 61 - 77

PIOTROWSKI, J.:

Tiergerechte Pferdehaltung: Mehrraum-Pferdeauslaufhaltung mit individueller Vorratsfutterung. In: Aktuelle Arbeiten zur artgemäßen Tierhaltung. KTBL, Darmstadt, Landwirtschaftsverlag GmbH, Münster-Hiltrup, 1989

PIRKELMANN, H.:

Pferdehaltung. In: Artgemäße und rentable Nutztierhaltung. Hrsg.: GRAUVOGL, A., Verlags Union Agrar, (1997), 123 - 159

RUCKEBUSCH, Y.:

The relevance of drowsiness in the circadian cycle of farm animals. Anim. Behav. 20 (1972), 637 - 643 
Arch. Tierz. 43 (2000) 4

\section{SAMBRAUS, H.H.:}

Ethologie der landwirtschaftlichen Nutztiere. Schweiz. Arch. Tierheilk. 117 (1975), 193 - 218

SCHÅFER, M.:

Spezielle Ethologie - Pferd. In: Nutztierethologie. Hrsg.: SAMBRAUS H.H., Paul Parey Verlag, Berlin, (1978), $214-245$

STEINHART, P.:

Der Schlaf des Pferdes. Univ. Munchen, Diss. med. vet., 1937

ZEITLER-FEICHT, M.H.:

Mindestanforderungen an die Gruppenhaltung von Pferden unter Tierschutzgesichtspunkten. Tierärztl.

Umschau 51 (1996), $611-614$

Eingegangen: 10.05 .2000

Akzeptiert: 29.06 .2000

Anschrif der Verfasser

Dr. MARGIT H. ZEITLER-FEICHT, Dipl. Ing. agr. VERENA PRANTNER

Lehrgebiet für Tierhaltung und Verhaltenskunde

Technische Universität München

D-85350 Freising-Weihenstephan 


\title{
Buchbesprechung
}

\author{
Pferdeinfluenza
}

WERNER LANGE

201 Seiten, 36 Abbildungen, 28 Tabellen, Parey Buchverlag Berlin, 2000, ISBN 3-8263-3321-7, 86,00 DM

Erkrankungen des Atmungsapparates gehören zu den am hăufigsten auftretenden Erkrankungen bei Pferden. Zweifelsfrei besitzen Influenzaviren bei Atemwegserkrankungen eine herausragende Bedeutung. Durch Influenza kann die Gesundheit und Leistungsfahigkeit von Pferden im erheblichen Maße beeintrăchtigt werden. Darüber hinaus entstehen durch die Erkrankung wirtschaftliche Verluste. Von diesen Tatsachen ausgehend ist es das Anliegen des Autors, insbesondere Tierärzten aber auch interessierten Züchtern und Trainern eine aktuelle Zusammenfassung des Wissens uber Pferdeinfluenza als Grundlage fulr eine erfolgreiche Bekämpfung zu geben. Die vorliegende Monographie wurde zu diesem Zweck in zwölf Kapitel gegliedert. Nach einer kurzen Einleitung folgen die Kapitel Virologie, Immunologie der Influenza, Diagnostik, Klinik der Influenza, Epidemiologie der Pferdeinfluenza, Ökologie der Influenza, Schutzimpfung, Chemotherapie und -prophylaxe, Überwachung der Pferdeinfluenza sowie Empfehlungen zur Bekămpfung der Pferdeinfluenza und ein Literaturverzeichnis. Ein Glossar und ein Sachwortverzeichnis bilden den Abschluss. Mit der Monographie ist es dem Autor durchgångig gelungen, den aktuellen Wissensstand in komprimierter Form und gleichzeitig gut verständlich niederzulegen. $\mathrm{Zu}$ diesem Ergebnis tragen die aussagefahigen und abersichtlichen Abbildungen ebenso bei wie die informativen Tabellen. Hervorzuheben ist die Tatsache, dass die wissenschaftlichen Aspekte der Thematik auf den Gebieten Virologie oder Immunologie der Influenza in sehr guter Weise mit den praktischen Aspekten der Krankheitsbekămpfung (Diagnostik, Prophylaxe und Therapie) verknupft wurden. Im Kapitel Okologie der Influenza erhält der Leser daruber hinaus einen interessanten Einblick in die Problematik von InterspeziesÜbertragungen. Zusammenfassend kann festgestellt werden, dass die Zielstellung, unter der die Monographie konzipiert wurde, in sehr guter Weise erreicht wurde. Mit der Monographie wird eine Lucke im deutschen Schrifttum zur Thematik geschlossen. Das Werk kann den interessierten Lesern sehr empfohlen werden.

WILHELM KANITZ, Dummerstorf 